\title{
UNIFORM APPROXIMATION OF CONTINUOUS MAPPINGS BY SMOOTH MAPPINGS WITH NO CRITICAL POINTS ON HILBERT MANIFOLDS
}

\author{
DANIEL AZAGRA AND MANUEL CEPEDELLO BOISO
}

\begin{abstract}
We prove that every continuous mapping from a separable infinitedimensional Hilbert space $X$ into $\mathbb{R}^{m}$ can be uniformly approximated by $C^{\infty}$ smooth mappings with no critical points. This kind of result can be regarded as a sort of very strong approximate version of the Morse-Sard theorem. Some consequences of the main theorem are as follows. Every two disjoint closed subsets of $X$ can be separated by a one-codimensional smooth manifold which is a level set of a smooth function with no critical points; this fact may be viewed as a nonlinear analogue of the geometrical version of the Hahn-Banach theorem. In particular, every closed set in $X$ can be uniformly approximated by open sets whose boundaries are $C^{\infty}$ smooth one-codimensional submanifolds of $X$. Finally, since every Hilbert manifold is diffeomorphic to an open subset of the Hilbert space, all of these results still hold if one replaces the Hilbert space $X$ with any smooth manifold $M$ modelled on $X$.
\end{abstract}

\section{INTRODUCTION AND MAIN RESULTS}

A fundamental result in differential topology and analysis is the Morse-Sard theorem [19, 20], which states that if $f: \mathbb{R}^{n} \longrightarrow \mathbb{R}^{m}$ is a $C^{r}$ smooth function, with $r>\max \{n-m, 0\}$, and $C_{f}$ stands for the set of critical points of $f$ (that is, the points $x$ at which the differential $d f(x)$ is not surjective), then the set of critical values, $f\left(C_{f}\right)$, is of (Lebesgue) measure zero in $\mathbb{R}^{m}$. This result also holds true for smooth functions $f: X \longrightarrow Y$ between two smooth manifolds of dimensions $n$ and $m$ respectively.

Several authors have dealt with the question as to what extent one can obtain a similar result for infinite-dimensional spaces or manifolds modelled on such spaces. Let us recall some of their results.

Smale [22 proved that if $X$ and $Y$ are separable connected smooth manifolds modelled on Banach spaces and $f: X \longrightarrow Y$ is a $C^{r}$ Fredholm map (that is, every differential $d f(x)$ is a Fredholm operator between the corresponding tangent spaces) then $f\left(C_{f}\right)$ is meager, and in particular $f\left(C_{f}\right)$ has no interior points, provided that $r>\max \{\operatorname{index}(d f(x)), 0\}$ for all $x \in X$; here index $(d f(x))$ stands for the index of the Fredholm operator $d f(x)$, that is, the difference between the dimension of the kernel

Date: March 22, 2002.

The first-named author was supported by a Marie Curie Fellowship of the European Community Training and Mobility of Researchers Programme under contract number HPMF-CT-2001-01175. 
of $d f(x)$ and the codimension of the image of $d f(x)$, which are both finite. However, these assumptions are quite restrictive: for instance, if $X$ is infinite-dimensional then there is no Fredholm map $f: X \longrightarrow \mathbb{R}$. In general, the existence of a Fredholm map $f$ from a manifold $X$ into another manifold $Y$ implies that $Y$ is infinite-dimensional whenever $X$ is.

On the other hand, one cannot dream of extending the Morse-Sard theorem to infinite dimensions without imposing strong restrictions. Indeed, as shown by Kupka's counterexample [16], there are $C^{\infty}$ smooth functions $f: X \longrightarrow \mathbb{R}$, where $X$ is a Hilbert space, so that their sets of critical values $f\left(C_{f}\right)$ contain intervals and in particular have non-empty interior.

More recently, S. M. Bates has carried out a deep study concerning the sharpness of the hypothesis of the Morse-Sard theorem and the geometry of the sets of critical values of smooth functions. In particular he has shown that the above $C^{r}$ smoothness hypothesis in the statement of the Morse-Sard theorem can be weakened to $C^{r-1,1}$; see [3, 4, 5, 6, 7]. C. G. Moreira and Bates have studied some generalizations of the Morse-Sard theorem related to Hausdorff measures and Hausdorff dimensions. They have also shown that the function $f$ as in Kukpa's counterexample can even be assumed to be a polymonial of degree three; see [8, 17].

Nevertheless, for many applications of the Morse-Sard theorem, it is often enough to know that any given continuous function can be uniformly approximated by a map whose set of critical values has empty interior. In this direction, Eells and McAlpin established the following theorem [14]: if $X$ is a separable Hilbert space, then every continuous function from $X$ into $\mathbb{R}$ can be uniformly approximated by a smooth function $f$ whose set of critical values $f\left(C_{f}\right)$ is of measure zero. This allowed them to deduce a version of this theorem for mappings between smooth manifolds $M$ and $N$ modelled on $X$ and a Banach space $F$ respectively, which they called an approximate Morse-Sard theorem: every continuous mapping from $M$ into $N$ can be uniformly approximated by a smooth function $f: M \longrightarrow N$ so that $f\left(C_{f}\right)$ has empty interior. However, this seemingly much more general version of the result is a bit tricky: indeed, as they already observed ([14], Remark 3A), when $F$ is infinitedimensional, the function $f$ they obtain satisfies that $C_{f}=M$, although $f(M)$ has empty interior in $N$. Unfortunately, even though all the results of that paper seem to be true, some of the proofs are not correct.

In this paper we will prove a much stronger result: if $M$ is a $C^{\infty}$ smooth manifold modelled on a separable infinite-dimensional Hilbert space $X$ (in the sequel such a manifold will be called a Hilbert manifold), then every continuous mapping from $M$ into $\mathbb{R}^{m}$ can be uniformly approximated by $C^{\infty}$ smooth mappings with no critical points. This kind of result might be regarded as the strongest possible one of any class of approximate Morse-Sard theorems, when the target space is finite-dimensional.

As a by-product we also obtain the following: for every open set $U$ in a separable Hilbert manifold $M$ there is a $C^{\infty}$ smooth function $f$ whose support is the closure of $U$ and so that $d f(x) \neq 0$ for every $x \in U$. This result could be summed up by saying that for every open subset $U$ of $M$ there is a function $f$ whose open support 
is $U$ and which does not satisfy Rolle's theorem; one should compare this result with the main theorem from [2] (see also the references therein).

Either of these results has in turn interesting consequences related to smooth approximation and separation of closed sets. For instance, every two disjoint closed subsets in $M$ can be separated by a smooth one-codimensional submanifold of $M$ which is a level set of a smooth function with no critical points. This may be regarded as a nonlinear analogue of the geometrical version of the Hahn-Banach theorem. In particular, every closed subset of $M$ can be uniformly approximated by open sets whose boundaries are smooth one-codimensional submanifolds of $M$.

So far these are some good consequences of our main result, all of them somehow related to Morse-Sard type theorems. But there are some bad consequences as well, perhaps the most noticeable one being that, since the set of smooth functions with no critical points is dense in the set of continuous functions defined on a Hilbert manifold, there are quite large sets of smooth functions for which no conceivable Morse theory could be valid.

Let us now formally state our main results. For the sake of a convenient notation in our proofs, when $\varphi$ takes real values we indistinctly use the symbols $d \varphi(x)=\varphi^{\prime}(x)$ to denote the derivative of $\varphi$ at a point $x$, and we reserve $d \varphi(x)$ for the derivative of a vector-valued function $\varphi: M \longrightarrow \mathbb{R}^{m}$ at a point $x \in M$.

Theorem 1.1. Let $U$ be an open subset of a separable infinite-dimensional Hilbert space $X$. Then, for every continuous mapping $f: U \longrightarrow \mathbb{R}^{m}$ and for every continuous positive function $\varepsilon: U \longrightarrow(0,+\infty)$, there exists a $C^{\infty}$ smooth mapping $\psi: U \longrightarrow \mathbb{R}^{m}$ such that $\|f(x)-\psi(x)\| \leq \varepsilon(x)$ and $d \psi(x)$ is surjective for all $x \in X$ (that is, $\psi$ has no critical points).

We will prove this result in the following section. Let us now establish the announced consequences of Theorem 1.1.

One could adapt the ideas in our proof to extend Theorem 1.1 to the setting of Hilbert manifolds but, for simplicity, we will instead use another approach. Indeed, bearing in mind a fundamental result on Hilbert manifolds due to Eells and Elworthy 113 that every separable Hilbert manifold can be $C^{\infty}$ embedded as an open subset of the Hilbert space, it is a triviality to observe that Theorem 1.1 still holds if we replace $U$ with a separable Hilbert manifold.

Theorem 1.2. Let $M$ be a separable Hilbert manifold. Then, for every continuous mapping $f: M \longrightarrow \mathbb{R}^{m}$ and every continuous positive function $\varepsilon: M \longrightarrow(0,+\infty)$, there exists a $C^{\infty}$ smooth mapping $\psi: M \longrightarrow \mathbb{R}^{m}$ such that $\psi$ has no critical points and $\|f(x)-\psi(x)\| \leq \varepsilon(x)$ for all $x \in X$.

Proof. According to the main theorem of [13], there is a $C^{\infty}$ embedding of $M$ onto an open subset of the Hilbert space $X$. Therefore $M$ is $C^{\infty}$ diffeomorphic to an open subset $U$ of $X$; let $h: U \longrightarrow M$ be such a $C^{\infty}$ diffeomorphism. Consider the continuous mappings $g=f \circ h: U \longrightarrow \mathbb{R}^{m}$ and $\delta=\varepsilon \circ h: U \longrightarrow(0,+\infty)$. By Theorem 1.1 there is a $C^{\infty}$ smooth function $\varphi: U \longrightarrow \mathbb{R}^{m}$ so that $\varphi$ has no critical 
points, and

$$
\|g(y)-\varphi(y)\| \leq \delta(y)
$$

for all $y \in U$. Now define $\psi=\varphi \circ h^{-1}: M \longrightarrow \mathbb{R}^{m}$. Since $h$ is a diffeomorphism it is clear that $h$ takes the critical set of $\psi$ onto the critical set of $\varphi=\psi \circ h$. But, as the latter is empty, so is the former; that is, $\psi$ has no critical points either. On the other hand, we have that

$$
\|f(x)-\psi(x)\|=\left\|g\left(h^{-1}(x)\right)-\varphi\left(h^{-1}(x)\right)\right\| \leq \delta\left(h^{-1}(x)\right)=\varepsilon(x)
$$

for all $x \in M$.

As an easy corollary we can deduce our promised nonlinear version of the geometrical Hahn-Banach theorem.

We will say that an open subset $U$ of a Hilbert manifold $M$ is smooth provided that its boundary $\partial U$ is a smooth one-codimensional submanifold of $M$.

Corollary 1.3. Let $M$ be a separable Hilbert manifold. Then, for every two disjoint closed subsets $C_{1}, C_{2}$ of $M$, there exists a $C^{\infty}$ smooth function $\varphi: M \longrightarrow \mathbb{R}$ with no critical points, such that the level set $N=\varphi^{-1}(0)$ is a 1-codimensional $C^{\infty}$ smooth submanifold of $M$ that separates $C_{1}$ and $C_{2}$, in the following sense. Define $U_{1}=\{x \in M: \varphi(x)<0\}$ and $U_{2}=\{x \in M: \varphi(x)>0\}$; then $U_{1}$ and $U_{2}$ are disjoint $C^{\infty}$ smooth open sets of $M$ with common boundary $\partial U_{1}=\partial U_{2}=N$, so that $C_{i} \subset U_{i}$ for $i=1,2$.

Proof. By Urysohn's lemma there exists a continuous function $f: M \longrightarrow[0,1]$ so that $C_{1} \subset f^{-1}(0)$ and $C_{2} \subset f^{-1}(1)$. Taking $\varepsilon=1 / 3$ and applying Theorem 1.2 we get a $C^{\infty}$ smooth function $\psi: M \longrightarrow \mathbb{R}$ which has no critical points and is so that

$$
|f(x)-\psi(x)| \leq 1 / 3
$$

for all $x \in M$; in particular

$$
C_{1} \subseteq f^{-1}(0) \subseteq \psi^{-1}(-\infty, 1 / 2):=U_{1},
$$

and

$$
C_{2} \subseteq f^{-1}(1) \subseteq \psi^{-1}(1 / 2,+\infty):=U_{2} .
$$

The open sets $U_{1}$ and $U_{2}$ are smooth because their common boundary $N=\psi^{-1}(1 / 2)$ is a smooth one-codimensional submanifold of $M$ (thanks to the implicit function theorem and the fact that $d \psi(x) \neq 0$ for all $x \in N)$. In order to obtain the result in the above form it is enough to set $\varphi=\psi-1 / 2$.

A trivial consequence of this result is that every closed subset of $M$ can be uniformly approximated by smooth open subsets of $M$. In fact,

Corollary 1.4. Every closed subset of a separable Hilbert manifold $M$ can be approximated by smooth open subsets of $M$, in the following sense: for every closed set $C \subset M$ and every open set $W$ containing $C$ there is a $C^{\infty}$ smooth open set $U$ so that $C \subset U \subseteq W$. 
Finally, the following result, which also implies the above corollary, tells us that for every open set $U$ in $M$ there always exists a function whose open support is $U$ and which does not satisfy Rolle's theorem.

Theorem 1.5. For every open subset $U$ of a Hilbert manifold $M$ there is a continuous function $f$ on $M$ whose support is the closure of $U$, so that $f$ is $C^{\infty}$ smooth on $U$ and yet $f$ has no critical point in $U$.

Proof. For the same reasons as in the proof of Theorem 1.2 we may assume that $U$ is an open subset of the Hilbert space $X=\ell_{2}$. Let $\varepsilon: X \longrightarrow[0,+\infty)$ be the distance function to $X \backslash U$, that is,

$$
\varepsilon(x)=\operatorname{dist}(x, X \backslash U)=\inf \{\|x-y\|: y \in X \backslash U\} .
$$

The function $\varepsilon$ is continuous on $X$ and satisfies that $\varepsilon(x)>0$ if and only if $x \in U$. According to Theorem 1.1, and setting $f(x)=2 \varepsilon(x)$, there exists a $C^{\infty}$ smooth function $\psi: U \longrightarrow \mathbb{R}$ which has no critical points on $U$, and such that $\varepsilon$-approximates $f$ on $U$, that is,

$$
|2 \varepsilon(x)-\psi(x)| \leq \varepsilon(x)
$$

for all $x \in U$. This inequality implies that

$$
\lim _{x \rightarrow z} \psi(x)=0
$$

for every $z \in \partial U$. Therefore, if we set $\psi=0$ on $X \backslash U$, the extended function $\psi: X \longrightarrow[0,+\infty)$ is continuous on the whole of $X$, is $C^{\infty}$ smooth on $U$ and has no critical points on $U$. On the other hand, $\psi(x) \geq \varepsilon(x)>0$ for all $x \in U$, hence the support of $\psi$ is $\bar{U}$.

\section{Proof of the MAIN RESUlT}

The main idea behind the proof of Theorem 1.1 is as follows. First we use a perturbed smooth partition of unity to approximate the given continuous mapping $f$. The summands of this perturbed partition of unity are functions supported on scalloped balls and carefully constructed in such a way that the critical set $C_{\varphi}$ of the approximating $\operatorname{sum} \varphi$ is locally compact.

Then we have to eliminate all the critical points without losing much of the approximation. To this end we compose the approximating mapping $\varphi$ with a deleting diffeomorphism $h: X \longrightarrow X \backslash C_{\varphi}$ which extracts the critical points $C_{\varphi}$ and is as close to the identity as we want. The existence of such a diffeomorphism is guaranteed by a quite elaborated result of West's [23]. In this way we obtain a smooth mapping $\psi$ which has no critical points, and which happens to approximate the function $\varphi$ (which in turn approximates the original $f$ ) because the perturbation brought on $\varphi$ by the composition with $h$ is not very important (recall that $h$ is arbitrarily closed to the identity).

The following proposition shows the existence of a function $\varphi$ with the above properties. Recall that $C_{\varphi}$ stands for the set of critical points of $\varphi$. 
Proposition 2.1. Let $U$ be an open subset of the separable Hilbert space $X$. Let $f: U \longrightarrow \mathbb{R}^{m}$ be a continuous mapping, and $\varepsilon: U \longrightarrow(0, \infty)$ a continuous positive function. Then there exist a $C^{\infty}$ smooth mapping $\varphi: U \longrightarrow \mathbb{R}^{m}$ so that

(a) $C_{\varphi}$ is locally compact and closed (relatively to $U$ );

(b) $\|\varphi(x)-f(x)\| \leq \varepsilon(x) / 2$ for all $x \in U$.

In fact, when the function $f$ takes values in the real line, we can obtain a much stronger result which is interesting in itself and might have some applications beyond the problem we are dealing with, as it provides much more accurate information about the structure and location of the critical points of the approximation $\varphi$. The following theorem shows that any continuous function can be uniformly approximated by $C^{\infty}$ smooth functions whose sets of critical points consist of countable union of compact sets which are separated by pairwise disjoint arbitrarily small open sets.

Theorem 2.2. Let $U$ be an open subset of the separable Hilbert space $X$. Let $f$ : $U \longrightarrow \mathbb{R}$ be a continuous function on $X$, and $\varepsilon: U \longrightarrow(0, \infty)$ a continuous positive function. Then there exist a $C^{\infty}$ smooth function $\varphi: U \longrightarrow \mathbb{R}$, sequences $\left(K_{n}\right)$ and $\left(U_{n}\right)$ of compact sets and open sets respectively, and a sequence $\left(B\left(y_{n}, r_{n}\right)\right)$ of open balls which are contained in $U$ and whose union covers $U$, such that:

(a) $C_{\varphi} \subseteq \bigcup_{n=1}^{\infty} K_{n}$;

(b) $K_{n} \subset U_{n} \subseteq B\left(y_{n}, r_{n}\right)$ for all $n$, and $U_{n} \cap U_{m}=\emptyset$ whenever $n \neq m$;

(c) $|\varphi(x)-f(x)| \leq \varepsilon(x)$ for all $x$, and $|\varphi(y)-f(x)| \leq \varepsilon\left(y_{n}\right)$ for every $x, y \in$ $B\left(y_{n}, r_{n}\right)$ and every $n$

(d) for every $x \in U$ there exists an open neighborhood $V_{x}$ of $x$ such that, either $V_{x}=U_{n}$ for a unique $n=n_{x}$, or else $V_{x} \cap U_{n}=\emptyset$ for all $n$.

Moreover, for any given $r>0$, the radii of the balls can be chosen so that $r_{n} \leq r$ for all $n$.

Finally, the following restatement of a striking result of West's [23] ensures the existence of the diffeomorphism $h$. We say that a mapping $g$ from a subset $A$ of $M$ is limited by an open cover $G$ of $M$ if the collection $\{\{x, g(x)\}: x \in A\}$ refines $G$.

Theorem 2.3 (West). Let $C$ be a closed, locally compact subset of a Hilbert manifold $M, U$ an open subset of $M$ with $C \subset U$, and $G$ an open cover of $M$. Then there is a $C^{\infty}$ diffeomorphism $h$ of $M$ onto $M \backslash C$ which is the identity outside $U$ and is limited by $G$.

Assume for a while that Proposition 2.1 is already established, and let us see how we can deduce Theorem 1.1.

\section{Proof of Theorem 1.1}

For the given continuous mappings $f$ and $\varepsilon$, take a mapping $\varphi$ with the properties of Proposition 2.1. Since $\varphi$ and $\varepsilon$ are continuous, for every $z \in U$ there exists $\delta_{z}>0$ so that if $x, y \in B\left(z, \delta_{z}\right)$ then

$$
\|\varphi(y)-\varphi(x)\| \leq \varepsilon(z) / 4 \leq \varepsilon(x) / 2 .
$$


Let $G=\left\{B\left(x, \delta_{x}\right): x \in U\right\}, M=U$, and for the critical set $C=C_{\varphi}$, use Theorem 2.3 to find a $C^{\infty}$ diffeomorphism $h: U \longrightarrow U \backslash C$ so that $h$ is limited by $G$. Define $\psi=\varphi \circ h$.

Since $h$ is limited by $G$ we have that, for any given $x \in U$, there exists $z \in U$ such that $x, h(x) \in B\left(z, \delta_{z}\right)$, and therefore $\|\varphi(h(x))-\varphi(x)\| \leq \varepsilon(z) / 4$, that is, we have that

$$
\|\psi(x)-\varphi(x)\| \leq \varepsilon(z) / 4 \leq \varepsilon(x) / 2 .
$$

Hence, by combining this inequality with (b) of Proposition 2.1, we obtain that

$$
\|\psi(x)-f(x)\| \leq \varepsilon(x)
$$

for all $x \in U$.

Let us see that $\psi$ does not have any critical point. The derivative of $\psi$ is given by

$$
d \psi(x)=d \varphi(h(x)) \circ d h(x) .
$$

Since $h(x) \notin C=C_{\varphi}$, we have that the linear map $d \varphi(h(x))$ is surjective. On the other hand $d h(x)$ is a linear isomorphism (because $h$ is a diffeomorphism). Then it is clear that the composition $d \psi(x)=d \varphi(h(x)) \circ d h(x)$ is a linear surjection from $X$ onto $\mathbb{R}^{m}$, for every $x \in U$.

Remark 2.4. In the case when $f: U \longrightarrow \mathbb{R}$ we do not need to use the full power of West's result. Thanks to the more accurate statement provided by Theorem 2.2 we can instead use a much more elementary result that tells us that for every compact subset $K$ and every open subset $U$ of $X$ with $K \subset U$, there exists a $C^{\infty}$ diffeomorphism $h: X \longrightarrow X \backslash K$ such that $h$ restricts to the identity outside $U$. In our case, to eliminate the critical points of the approximating function $\varphi$ of Theorem 2.2, we may compose $\varphi$ with a sequence of deleting diffeomorphisms $h_{n}: X \longrightarrow X \backslash K_{n}$ which extract each of the compact sets of critical points $K_{n}$ and restrict to the identity outside each of the open sets $U_{n}$. The infinite composition of deleting diffeomorphisms with our function, $\psi=\varphi \circ \bigcirc_{n=1}^{\infty} h_{n}$, is locally finite, in the sense that only a finite number (in fact at most one) of the diffeomorphisms are acting on some neighborhood of each point, while all the rest restrict to the identity on that neighborhood. As in the proof above, it follows that $\psi$ has no critical points (we can use exactly the same argument locally), and still approximates $f$ (recall that each $h_{n}$ restricts to the identity outside the set $U_{n}$, on which $\varphi$ has a very small oscillation, and the $U_{n}$ are pairwise disjoint).

\section{Proof of Proposition 2.1}

We will assume that $U=X$, since the proof is completely analogous in the case of a general open set. One only has to take some (easy but rather rambling) technical precautions in order to make sure that the different balls considered in the argument are in $U$.

In order to avoid bearing an unnecessary burden of notation, we will make the proof of this proposition for the case of a constant $\varepsilon>0$. Later on we will briefly 
explain what additional technical precautions must be taken in order to deduce the general form of this result (see Remark 2.11 below).

Let $B(x, r)$ and $\bar{B}(x, r)$ stand for the open ball and closed ball, respectively, of center $x$ and radius $r$, with respect to the usual hilbertian norm $\|\cdot\|$ of $X$.

Case I. We will first consider the case of a real valued function $f: U \longrightarrow \mathbb{R}$. Fix $\varepsilon>0$. By continuity, for every $x \in X$ there exists $\delta_{x}>0$ so that $|f(y)-f(x)| \leq \varepsilon / 8$ whenever $y \in B\left(x, 2 \delta_{x}\right)$. Since $X=\bigcup_{x \in X} B\left(x, \delta_{x} / 2\right)$ is separable, there exists a countable subcovering,

$$
X=\bigcup_{n=1}^{\infty} B\left(x_{n}, r_{n} / 2\right),
$$

where $r_{n}=\delta_{x_{n}}$, for some sequence of centers $\left(x_{n}\right)$. By induction (and using the fact that every finite-dimensional subspace of $X$ has empty interior in $X$ ), we can choose a sequence of linearly independent vectors $\left(y_{n}\right)$, with $y_{n} \in B\left(x_{n}, r_{n} / 2\right)$, so that

$$
X=\bigcup_{n=1}^{\infty} B\left(y_{n}, r_{n}\right) \text {. }
$$

Moreover, we have that

$$
\left|f(y)-f\left(y_{n}\right)\right| \leq \varepsilon / 4 \text { whenever }\left\|y-y_{n}\right\| \leq r_{n} .
$$

Now we define the scalloped balls $B_{n}$ that are the basis for our perturbed partition of unity: set $B_{1}=B\left(y_{1}, r_{1}\right)$, and for $n \geq 2$ define

$$
B_{n}=B\left(y_{n}, r_{n}\right) \backslash\left(\bigcup_{j=1}^{n-1} \bar{B}\left(y_{j}, \lambda_{n} r_{j}\right)\right) ;
$$

where $1 / 2<\lambda_{2}<\lambda_{3}<\ldots<\lambda_{n}<\lambda_{n+1}<\ldots<1$, with $\lim _{n \rightarrow \infty} \lambda_{n}=1$.

Taking into account that $\lim _{n \rightarrow \infty} \lambda_{n}=1$, it is easily checked that the $B_{n}$ form a locally finite open covering of $X$, with the nice property that

$$
\left|f(y)-f\left(y_{n}\right)\right| \leq \varepsilon / 4 \text { whenever } y \in B_{n} .
$$

Next, pick a $C^{\infty}$ smooth function $g_{1}: \mathbb{R} \longrightarrow[0,1]$ so that:

(i) $g_{1}(t)=1$ for $t \leq 0$,

(ii) $g_{1}(t)=0$ for $t \geq r_{1}^{2}$,

(iii) $g_{1}^{\prime}(t)<0$ if $0<t<r_{1}^{2}$;

and define then $\varphi_{1}: X \longrightarrow \mathbb{R}$ by

$$
\varphi_{1}(x)=g_{1}\left(\left\|x-y_{1}\right\|^{2}\right)
$$

for all $x \in X$. Note that $\varphi_{1}$ is a $C^{\infty}$ smooth function whose open support is $B_{1}$, and $B_{1} \cap C_{\varphi_{1}}=\left\{y_{1}\right\}$, that is, $y_{1}$ is the only critical point of $\varphi_{1}$ that lies inside $B_{1}$.

Now, for $n \geq 2$, pick $C^{\infty}$ smooth functions $\theta_{(n, j)}: \mathbb{R} \longrightarrow[0,1], j=1, \ldots, n$, with the following properties. For $j=1, \ldots, n-1, \theta_{(n, j)}$ satisfies that

(i) $\theta_{(n, j)}(t)=0$ for $t \leq\left(\lambda_{n} r_{j}\right)^{2}$,

(ii) $\theta_{(n, j)}(t)=1$ for $t \geq r_{j}^{2}$, 
(iii) $\theta_{(n, j)}^{\prime}(t)>0$ if $\left(\lambda_{n} r_{j}\right)^{2}<t<r_{j}^{2}$;

while for $j=n$ the function $\theta_{(n, n)}$ is such that

(i) $\theta_{(n, n)}(t)=1$ for $t \leq 0$,

(ii) $\theta_{(n, n)}(t)=0$ for $t \geq r_{n}^{2}$,

(iii) $\theta_{(n, n)}^{\prime}(t)<0$ if $0<t<r_{n}^{2}$.

Then define the function $g_{n}: \mathbb{R}^{n} \longrightarrow[0,1]$ as

$$
g_{n}\left(t_{1}, \ldots, t_{n}\right)=\prod_{i=1}^{n} \theta_{(n, i)}\left(t_{i}\right)
$$

for all $t=\left(t_{1}, \ldots, t_{n}\right) \in \mathbb{R}^{n}$. This function is clearly $C^{\infty}$ smooth on $\mathbb{R}^{n}$ and satisfies the following properties:

(i) $g_{n}\left(t_{1}, \ldots, t_{n}\right)>0$ if and only if $t_{j}>\left(\lambda_{n} r_{j}\right)^{2}$ for all $j=1, \ldots, n-1$, and $t_{n}<r_{n}{ }^{2}$; and $g_{n}$ vanishes elsewhere;

(ii) $g_{n}\left(t_{1}, \ldots, t_{n}\right)=\theta_{(n, n)}\left(t_{n}\right)$ whenever $t_{j} \geq r_{j}{ }^{2}$ for all $j=1, \ldots, n-1$;

(iii) $\nabla g_{n}\left(t_{1}, \ldots, t_{n}\right) \neq 0$ provided $\left(\lambda_{n} r_{j}\right)^{2}<t_{j}$ for all $j=1, \ldots, n-1$, and $0<t_{n}<$ $r_{n}{ }^{2}$.

Moreover, under the same conditions as in (iii) just above we have that

$$
\frac{\partial g_{n}}{\partial t_{n}}\left(t_{1}, \ldots, t_{n}\right)=\frac{\partial \theta_{(n, n)}}{\partial t_{n}}\left(t_{n}\right) \prod_{i=1}^{n-1} \theta_{(n, i)}\left(t_{i}\right)<0,
$$

since no function in this product vanishes on the specified set, while for $j<n$, according to the corresponding properties of the functions $\theta_{(n, j)}$ we have that

$$
\frac{\partial g_{n}}{\partial t_{j}}\left(t_{1}, \ldots, t_{n}\right)=\frac{\partial \theta_{(n, j)}}{\partial t_{j}}\left(t_{j}\right) \prod_{i=1, i \neq j}^{n} \theta_{(n, i)}\left(t_{i}\right)>0 .
$$

If we are not in the conditions of (iii) then the corresponding inequalities do still hold but are not strict.

Let us now define $\varphi_{n}: X \longrightarrow[0,1]$ by

$$
\varphi_{n}(x)=g_{n}\left(\left\|x-y_{1}\right\|^{2}, \ldots,\left\|x-y_{n}\right\|^{2}\right) .
$$

It is clear that $\varphi_{n}$ is a $C^{\infty}$ smooth function whose open support is precisely the scalloped ball $B_{n}$.

As above, let us denote by $C_{\varphi_{n}}$ the critical set of $\varphi_{n}$, that is,

$$
C_{\varphi_{n}}=\left\{x \in X: \varphi_{n}^{\prime}(x)=0\right\} .
$$

Since our norm $\|\cdot\|$ is hilbertian we have that, if $x \in C_{\varphi_{n}} \cap B_{n}$, then $x$ belongs to the affine span of $y_{1}, \ldots, y_{n}$. Indeed, if $x \in B_{n}$,

$$
\varphi_{n}^{\prime}(x)=\sum_{j=1}^{n} \frac{\partial g_{n}}{\partial t_{j}}\left(\left\|x-y_{1}\right\|^{2}, \ldots,\left\|x-y_{n}\right\|^{2}\right) 2\left(x-y_{j}\right)=0
$$


which (taking into account (5) and the fact that the $y_{j}$ are all linearly independent) means that $x$ is in the affine span of $y_{1}, \ldots, y_{n}$. Here, as is usual, we identify the Hilbert space $X$ with its dual $X^{*}$, and we make use of the fact that the derivative of the function $x \mapsto\|x\|^{2}$ is the mapping $x \mapsto 2 x$.

Similarly, by using (5) it can be shown that $x \in C_{\varphi_{1}+\cdots+\varphi_{m}} \cap\left(B_{1} \cup \ldots \cup B_{m}\right)$ implies that $x$ belongs to the affine span of $y_{1}, \ldots, y_{m}$.

In order that our approximating function has a small critical set we cannot use the standard approximation provided by the partition of unity associated with the functions $\left(\varphi_{j}\right)_{i \in \mathbb{N}}$, namely

$$
x \mapsto \frac{\sum_{n=1}^{\infty} \alpha_{n} \varphi_{n}(x)}{\sum_{n=1}^{\infty} \varphi_{n}(x)},
$$

where $\alpha_{n}=f\left(y_{n}\right)$. Indeed, such a function would have a huge set of critical points since it would be constant (equal to $\alpha_{n}$ ) on a lot of large places (at least on each $B_{n}$ minus the union of the rest of the $B_{j}$ ). Instead, we will modify this standard approximation by letting the $\alpha_{n}$ be functions (and not mere numbers) of very small oscillation and with only one critical point (namely $y_{n}$ ). So, for every $n \in \mathbb{N}$ let us pick a $C^{\infty}$ smooth real function $a_{n}:[0,+\infty) \longrightarrow \mathbb{R}$ with the following properties:

(i) $a_{n}(0)=f\left(y_{n}\right)$;

(ii) $a_{n}^{\prime}(t)<0$ whenever $t>0$;

(iii) $\left|a_{n}(t)-a_{n}(0)\right| \leq \varepsilon / 4$ for all $t \geq 0$;

and define $\alpha_{n}: X \longrightarrow \mathbb{R}$ by

$$
\alpha_{n}(x)=a_{n}\left(\left\|x-y_{n}\right\|^{2}\right)
$$

for every $x \in X$. It is clear that $\alpha_{n}$ is a $C^{\infty}$ smooth function on $X$ whose only critical point is $y_{n}$. Besides,

$$
\left|\alpha_{n}(x)-f\left(y_{n}\right)\right| \leq \varepsilon / 4 \text { for all } x \in X .
$$

Now we can define our approximating function $\varphi: X \longrightarrow \mathbb{R}$ by

$$
\varphi(x)=\frac{\sum_{n=1}^{\infty} \alpha_{n}(x) \varphi_{n}(x)}{\sum_{n=1}^{\infty} \varphi_{n}(x)}
$$

for every $x \in X$. Since the sums are locally finite, it is clear that $\varphi$ is a well-defined $C^{\infty}$ smooth function.

Fact 2.5. The function $\varphi$ approximates $f$ nicely. Namely, we have that

(i) $|\varphi(x)-f(x)| \leq \varepsilon / 2$ for all $x \in X$, and

(ii) $|\varphi(y)-f(x)| \leq \varepsilon$ for all $x, y \in B\left(y_{n}, r_{n}\right)$ and each $n \in \mathbb{N}$.

Proof. For every $n$ we have that $\left|\alpha_{n}(x)-f\left(y_{n}\right)\right| \leq \varepsilon / 4$ for all $x \in X$. On the other hand, by (4) above we know that $\left|f(x)-f\left(y_{n}\right)\right| \leq \varepsilon / 4$ whenever $x \in B\left(y_{n}, r_{n}\right)$. Then, by the triangle inequality, it follows that

$$
\left|\alpha_{n}(x)-f(x)\right| \leq \varepsilon / 2
$$


whenever $x \in B\left(y_{n}, r_{n}\right)$. In the same way we deduce that

$$
\left|\alpha_{m}(x)-f\left(y_{n}\right)\right| \leq \varepsilon / 2
$$

whenever $x \in B\left(y_{n}, r_{n}\right) \cap B\left(y_{m}, r_{m}\right)$. Since $\varphi_{m}(y)=0$ when $y \notin B\left(y_{m}, r_{m}\right)$, from (8) we get that

$$
|\varphi(x)-f(x)|=\left|\frac{\sum_{m=1}^{\infty}\left(\alpha_{m}(x)-f(x)\right) \varphi_{m}(x)}{\sum_{m=1}^{\infty} \varphi_{m}(x)}\right| \leq \frac{\sum_{m=1}^{\infty} \frac{\varepsilon}{2} \varphi_{m}(x)}{\sum_{m=1}^{\infty} \varphi_{m}(x)}=\varepsilon / 2
$$

for all $x \in X$, which shows $(i)$. Similarly, we deduce from (9) that

$$
\left|\varphi(y)-f\left(y_{n}\right)\right|=\left|\frac{\sum_{m=1}^{\infty}\left(\alpha_{m}(y)-f\left(y_{n}\right)\right) \varphi_{m}(y)}{\sum_{m=1}^{\infty} \varphi_{m}(y)}\right| \leq \frac{\sum_{m=1}^{\infty} \frac{\varepsilon}{2} \varphi_{m}(y)}{\sum_{m=1}^{\infty} \varphi_{m}(y)}=\varepsilon / 2
$$

for every $y \in B\left(y_{n}, r_{n}\right)$, which, combined with (4) above, yields that

$$
|\varphi(y)-f(x)| \leq \varepsilon / 2+\varepsilon / 4,
$$

for every $x, y \in B\left(y_{n}, r_{n}\right)$, so $(i i)$ is satisfied as well.

Now let us have a look at the derivative of $\varphi$. To this end let us introduce the auxiliary functions $f_{n}$ defined by

$$
f_{n}(x)=\frac{\sum_{k=1}^{n} \alpha_{k}(x) \varphi_{k}(x)}{\sum_{k=1}^{n} \varphi_{k}(x)}, \text { for all } x \in \bigcup_{i=1}^{n} B_{i} .
$$

Notice that $\varphi$ can be expressed as

$$
\varphi(x)=\lim _{n \rightarrow \infty} f_{n}(x),
$$

that the domains of the $f_{n}$ form an increasing tower of open sets whose union is $X$, and that each $f_{n}$ restricts to $f_{n-1}$ on $\bigcup_{i=1}^{n-1} B_{i} \backslash B_{n}$. Moreover, we have the following.

Fact 2.6. For each $x \in X$ there is an open neighborhood $V_{x}$ of $x$ and some $n_{x} \in \mathbb{N}$ so that $\varphi(y)=f_{n}(y)$ for all $y \in V_{x}$ and all $n \geq n_{x}$.

Proof. Indeed, we have that, for every $n \in \mathbb{N}$,

$$
\varphi(y)=f_{k}(y) \text { for all } y \in V_{n}:=\left(\bigcup_{j=1}^{n} B_{j}\right) \backslash\left(\bigcup_{i=n+1}^{\infty} \bar{B}_{i}\right), \text { and for all } k \geq n .
$$

The $V_{n}$ are open, $V_{n} \subseteq V_{n+1}$, and $\bigcup_{i=1}^{\infty} V_{i}=X$, because the covering of $X$ formed by the $B_{j}$ is locally finite.

Hence, by looking at the derivatives of the functions $f_{n}$ we will get enough information about the derivative of $\varphi$.

If $x \in \bigcup_{j=1}^{n} B_{j}$ then the expression for the derivative of $f_{n}$ is given by

$f_{n}^{\prime}(x)=\frac{\sum_{j=1}^{n}\left[\alpha_{j}^{\prime}(x) \varphi_{j}(x)+\alpha_{j}(x) \varphi_{j}^{\prime}(x)\right] \sum_{i=1}^{n} \varphi_{i}(x)-\sum_{j=1}^{n} \varphi_{j}^{\prime}(x) \sum_{i=1}^{n} \alpha_{i}(x) \varphi_{i}(x)}{\left(\sum_{j=1}^{n} \varphi_{j}(x)\right)^{2}}$. 
Therefore, for $x \in \bigcup_{j=1}^{n} B_{j}$ we have that $f_{n}^{\prime}(x)=0$ if and only if

$$
\sum_{j=1}^{n} \sum_{i=1}^{n} \varphi_{i}(x)\left[\alpha_{j}^{\prime}(x) \varphi_{j}(x)+\left(\alpha_{j}(x)-\alpha_{i}(x)\right) \varphi_{j}^{\prime}(x)\right]=0 .
$$

By inserting the expressions for the derivatives of $\varphi_{j}$ and $\alpha_{j}$ in equation (10), we can express the condition $f_{n}^{\prime}(x)=0$ as a nontrivial linear dependence link on the vectors $\left(x-y_{j}\right)$, which yields that $x$ is in the affine span of the points $y_{1}, \ldots, y_{n}$.

Notation 2.7. In the sequel $\mathcal{A}\left[z_{1}, \ldots, z_{k}\right]$ stands for the affine subspace spanned by a finite sequence of points $z_{1}, \ldots, z_{k} \in X$.

Fact 2.8. If $x \in C_{f_{n}} \cap B_{n}$, then $x \in \mathcal{A}_{n}:=\mathcal{A}\left[y_{1}, \ldots, y_{n}\right]$. Moreover, for each $n \in \mathbb{N}$ and for every finite sequence of positive integers $k_{1}<k_{2}<\ldots<k_{m}<n$ we have that

$$
C_{f_{n}} \cap\left(B_{n} \backslash \bigcup_{j=1}^{m} B_{k_{j}}\right) \subseteq \mathcal{A}\left[\left\{y_{1}, \ldots, y_{n}\right\} \backslash\left\{y_{k_{1}}, \ldots, y_{k_{m}}\right\}\right] .
$$

Proof. As above, in all the subsequent calculations, we will identify the Hilbert space $X$ with its dual $X^{*}$, and the derivative of $\|\cdot\|^{2}$ with the mapping $x \mapsto 2 x$. To save notation, let us simply write

$$
\frac{\partial g_{n}}{\partial t_{j}}\left(\left\|x-y_{1}\right\|^{2}, \ldots,\left\|x-y_{n}\right\|^{2}\right)=\mu_{(n, j)}
$$

and

$$
a_{j}^{\prime}\left(\left\|x-y_{j}\right\|^{2}\right)=\eta_{j}
$$

Notice that, according to (5) and (6) above, $\mu_{(n, j)} \geq 0$ for $j=1, \ldots, n-1$, while $\mu_{(n, n)} \leq 0$; and $\mu_{(n, n)} \neq 0$ provided $x \in B_{n}$ and $x \neq y_{n}$; on the other hand it is clear that $\eta_{j}<0$ for all $j$ unless $x=y_{j}$ (in which case $\eta_{j}=0$ ).

Assuming $x \in C_{f_{n}} \cap B_{n}$, and taking into account the expression (10) for $\varphi_{j}^{\prime}(x)$ and the fact that $\alpha_{j}^{\prime}(x)=2 \eta_{j}\left(x-y_{j}\right)$, we can write condition (10) above in the form

$$
2 \sum_{j=1}^{n} \sum_{i=1}^{n} \varphi_{i}(x)\left[\eta_{j} \varphi_{j}(x)\left(x-y_{j}\right)+\left(\alpha_{j}(x)-\alpha_{i}(x)\right) \sum_{\ell=1}^{j} \mu_{(j, \ell)}\left(x-y_{\ell}\right)\right]=0,
$$

which in turn is equivalent (taking the common factors of each $\left(x-y_{j}\right)$ together) to the following one

$$
\sum_{j=1}^{n}\left[\eta_{j} \varphi_{j}(x) \sum_{i=1}^{n} \varphi_{i}(x)+\sum_{k=j}^{n}\left(\sum_{i=1}^{n}\left(\alpha_{k}(x)-\alpha_{i}(x)\right) \varphi_{i}(x)\right) \mu_{(k, j)}\right]\left(x-y_{j}\right)=0 .
$$

Now notice that, if we can prove that at least one of the expressions multiplying the $\left(x-y_{j}\right)$ does not vanish then we are done; indeed, we will have that the vectors $x-y_{1}, \ldots, x-y_{n}$ are linearly dependent, which means that $x$ belongs to the affine span of the points $y_{1}, \ldots, y_{n}$. 
So let us check that not all of those expressions in (11) vanish. In fact we are going to see that at least one of the terms is strictly negative. We can obviously assume that $x$ is not any of the points $y_{1}, \ldots, y_{n}$ (which are already in $\mathcal{A}_{n}$ ). In this case we have that $\mu_{(n, n)}<0$ and $\eta_{j}<0$ for all $j=1, \ldots, n$. For simplicity, we will only make the argument in the case $n=3$; giving a proof in a more general case would be as little instructive as tedious to read.

Let us first assume that $\varphi_{j}(x) \neq 0$ for $j=1,2,3$. We begin by looking at the term that multiplies $\left(x-y_{3}\right)$ in $(11)$, that is

$$
\beta_{3}:=\eta_{3} \varphi_{3}(x) \sum_{i=1}^{3} \varphi_{i}(x)+\sum_{i=1}^{3}\left(\alpha_{3}(x)-\alpha_{i}(x)\right) \varphi_{i}(x) \mu_{(3,3)} .
$$

If $\sum_{i=1}^{3}\left(\alpha_{3}(x)-\alpha_{i}(x)\right) \varphi_{i}(x) \geq 0$ we are done, since in this case we easily see that $\beta_{3}<0$ (remember that $\mu_{(3,3)} \leq 0, \eta_{3}<0$, and $\varphi_{3}(x)>0$ ). Otherwise we have that

$$
\sum_{i=1}^{3}\left(\alpha_{3}(x)-\alpha_{i}(x)\right) \varphi_{i}(x)<0
$$

and then we look at the term $\beta_{2}$ multiplying $\left(x-y_{2}\right)$ in (11), namely,

$$
\beta_{2}:=\eta_{2} \varphi_{2}(x) \sum_{i=1}^{3} \varphi_{i}(x)+\sum_{k=2}^{3}\left(\sum_{i=1}^{3}\left(\alpha_{k}(x)-\alpha_{i}(x)\right) \varphi_{i}(x)\right) \mu_{(k, 2)} .
$$

Now, since $\mu_{(3,2)} \geq 0$, we have $\sum_{i=1}^{3}\left(\alpha_{3}(x)-\alpha_{i}(x)\right) \varphi_{i}(x) \mu_{(3,2)} \leq 0$, and on the other hand $\eta_{2} \varphi_{2}(x) \sum_{i=1}^{3} \varphi_{i}(x)<0$ so that, if $\sum_{i=1}^{3}\left(\alpha_{2}(x)-\alpha_{i}(x)\right) \varphi_{i}(x)$ happens to be nonnegative, then we also have $\sum_{i=1}^{3}\left(\alpha_{2}(x)-\alpha_{i}(x)\right) \varphi_{i}(x) \mu_{(2,2)} \leq 0$, and then we are done since $\beta_{2}$, being a sum of negative terms (one of them strictly negative) must be negative as well. Otherwise,

$$
\sum_{i=1}^{3}\left(\alpha_{2}(x)-\alpha_{i}(x)\right) \varphi_{i}(x)
$$

is negative, and then we finally pass to the term $\beta_{1}$ multiplying $\left(x-y_{1}\right)$ in $(11)$, that is,

$$
\beta_{1}:=\eta_{1} \varphi_{1}(x) \sum_{i=1}^{3} \varphi_{i}(x)+\sum_{k=1}^{3}\left(\sum_{i=1}^{3}\left(\alpha_{k}(x)-\alpha_{i}(x)\right) \varphi_{i}(x)\right) \mu_{(k, 1)} .
$$

Here, by the assumptions we have made so far and taking into account the signs of $\mu_{(k, j)}$ and $\eta_{j}$, we see that $\sum_{i=1}^{3}\left(\alpha_{k}(x)-\alpha_{i}(x)\right) \varphi_{i}(x) \mu_{(k, 1)} \leq 0$ for $k=2,3$. Having arrived at this point, it is sure that $\sum_{i=1}^{3}\left(\alpha_{1}(x)-\alpha_{i}(x)\right) \varphi_{i}(x)$ must be nonnegative (otherwise the numbers $\sum_{i=1}^{3}\left(\alpha_{k}(x)-\alpha_{i}(x)\right) \varphi_{i}(x)$ should be strictly negative for all $k=1,2,3$, which is impossible if one takes $\alpha_{k}(x)$ to be the maximum of the $\alpha_{i}(x)$ ), and now we can deduce as before that $\beta_{1}<0$.

Finally let us consider the case when some of the $\varphi_{i}(x)$ vanish, for $i=1,2$ (remember that $\varphi_{3}(x) \neq 0$ since $x \in B_{3}$, the open support of $\varphi_{3}$ ). From the 
definitions of $\mu_{(k, j)}, g_{n}$ and $\varphi_{n}$, it is clear that $\mu_{(k, j)}=0$ whenever $\varphi_{j}(x)=0$ or $\varphi_{k}(x)=0$, and bearing this fact in mind we can simplify equality (11) to a great extent by dropping all the terms that now vanish.

If $\varphi_{1}(x)=\varphi_{2}(x)=0$ then (11) reads

$$
\varphi_{3}(x)^{2} \eta_{3}\left(x-y_{3}\right)=0
$$

which cannot happen since we assumed $x \neq y_{j}$ (this means that the only critical point that $f_{n}$ can have in $B_{3} \backslash\left(B_{1} \cup B_{2}\right)$ is $\left.y_{3}\right)$.

If $\varphi_{1}(x)=0$ and $\varphi_{2}(x) \neq 0$ then the term $\beta_{1}$ accompanying $\left(x-y_{1}\right)$ in (11) vanishes, and hence (11) is reduced to

$$
\sum_{j=2}^{3}\left[\eta_{j} \varphi_{j}(x) \sum_{i=2}^{3} \varphi_{i}(x)+\sum_{k=j}^{3}\left(\sum_{i=2}^{3}\left(\alpha_{k}(x)-\alpha_{i}(x)\right) \varphi_{i}(x)\right) \mu_{(k, j)}\right]\left(x-y_{j}\right)=0 .
$$

Since at least one of the numbers $\sum_{i=2}^{3}\left(\alpha_{k}(x)-\alpha_{i}(x)\right) \varphi_{i}(x), k=2,3$, is nonnegative, the same reasoning as in the first case allows us to conclude that either $\beta_{3}$ or $\beta_{2}$ is strictly negative. Finally, in the case $\varphi_{1}(x) \neq 0$ and $\varphi_{2}(x)=0$, it is $\beta_{2}$ that vanishes, and (11) reads $\beta_{1}\left(x-y_{1}\right)+\beta_{3}\left(x-y_{3}\right)=0$, where

$$
\beta_{3}=\eta_{3} \varphi_{3}(x) \sum_{i=1, i \neq 2}^{3} \varphi_{i}(x)+\sum_{i=1, i \neq 2}^{3}\left(\alpha_{3}(x)-\alpha_{i}(x)\right) \varphi_{i}(x) \mu_{(3,3)},
$$

and

$$
\beta_{1}=\eta_{1} \varphi_{1}(x) \sum_{i=1, i \neq 2}^{3} \varphi_{i}(x)+\sum_{k=1, i \neq 2}^{3} \sum_{i=1, i \neq 2}^{3}\left(\alpha_{k}(x)-\alpha_{i}(x)\right) \varphi_{i}(x) \mu_{(k, 1)} .
$$

Again, at least one of the numbers $\sum_{i=1, i \neq 2}^{3}\left(\alpha_{k}(x)-\alpha_{i}(x)\right) \varphi_{i}(x), k=1,3$, is nonnegative, and the same argument as above applies.

Finally, bearing in mind the definition of the functions $\varphi_{j}$, whose open support are the $B_{j}$, it is clear that the above discussion shows, in fact, the following inclusions:

$$
\begin{aligned}
& C_{f_{3}} \cap B_{3} \subseteq \mathcal{A}\left[y_{1}, y_{2}, y_{3}\right] ; \\
& C_{f_{3}} \cap\left(B_{3} \backslash B_{1}\right) \subseteq \mathcal{A}\left[y_{2}, y_{3}\right], \text { and } C_{f_{3}} \cap\left(B_{3} \backslash B_{2}\right) \subseteq \mathcal{A}\left[y_{1}, y_{3}\right] ; \\
& C_{f_{3}} \cap\left(B_{3} \backslash\left(B_{1} \cup B_{2}\right)\right) \subseteq \mathcal{A}\left[y_{3}\right] .
\end{aligned}
$$

An analogous argument in the case $n \geq 4$ proves the second part of the statement of Fact 2.8.

Remark 2.9. Notice that the above proof shows that the derivative $d f_{n}(x)$ of the function $f_{n}$ at a point $x$ can be expressed as a nontrivial linear combination of the linear functionals $\left(x-y_{k}\right) \in \ell_{2}^{*}=\ell_{2}, k=1, \ldots, n$. That is, for every $x \in \bigcup_{i=1}^{n} B_{i}$ there are numbers $\beta_{1}(x), \ldots, \beta_{n}(x)$ such that at least one of them does not vanish, and

$$
d f_{n}(x)=\frac{1}{\left(\sum_{j=1}^{n} \varphi_{j}(x)\right)^{2}} \sum_{k=1}^{n} \beta_{k}(x)\left(x-y_{k}\right) .
$$


This will turn out to be a crucial observation when dealing with the case $m \geq 1$.

Since $\varphi$ has a continuous derivative, it is obvious that its critical set $C_{\varphi}$ is closed in $U$. According to Fact 2.6, $\varphi$ locally coincides with one of the $f_{n}$. From Fact 2.8 it follows that the set of critical points $C_{f_{n}}$ of each function $f_{n}$ is contained in a finite dimensional affine subspace of $X$. Therefore it is clear that the set $C_{\varphi}$ of critical points of $\varphi$ is locally contained in finite dimensional subspaces, that is, for each $x \in C_{\varphi}$ there is an open bounded neighborhood $V_{x}$ of $x$ so that $C_{\varphi} \cap \overline{V_{x}}$ is contained in a finite dimensional subspace $F_{x}$ of $X$ and hence is compact (as is closed and bounded as well). This means that $C_{\varphi}$ is locally compact, and concludes the proof of Proposition 2.1 in the case $m=1$.

Case II. Let us now deal with the case when $f: X \longrightarrow \mathbb{R}^{m}$ with $m \geq 2$. We denote $f=\left(f^{1}, \ldots, f^{m}\right)$, where $f^{1}, \ldots, f^{m}$ are the coordinate functions of $f$. In this case we have to construct $C^{\infty}$ smooth functions $\varphi^{1}, \ldots, \varphi^{m}$ so that each $\varphi^{j}$ uniformly approximates $f^{j}$ and the set of points $x \in X$ at which the derivatives $d \varphi^{1}(x), \ldots$, $d \varphi^{m}(x)$ are linearly dependent is locally compact. If we succeed in doing so then it is clear that the function $\varphi=\left(\varphi^{1}, \ldots, \varphi^{m}\right): X \longrightarrow \mathbb{R}^{m}$ will approximate $f$ and its set $C_{\varphi}$ of critical points will be closed and locally compact.

Let us define $\varepsilon_{j}=\varepsilon / \sqrt{4 m}, j=1, \ldots, m$. As each of the functions $f^{j}$, with $j=1, \ldots, m$, is continuous, for every $x \in X$ there exists $\delta_{x}^{j}>0$ so that

$$
\left|f^{j}(y)-f^{j}(x)\right| \leq \varepsilon_{j} / 8 \text { for all } y \in B\left(x, 2 \delta_{x}\right) .
$$

Since $X=\bigcup_{x \in X} B\left(x, \delta_{x}^{j} / 2\right)$ is separable, we may take a countable subcovering,

$$
X=\bigcup_{n=1}^{\infty} B\left(x_{n}^{j}, r_{n}^{j} / 2\right),
$$

where $r_{n}^{j}=\delta_{x_{n}}^{j}$, for each $j=1, \ldots, m$.

Now, we can slightly perturb the centers $x_{n}^{j}$ of the balls so that the union of all the $m$ sequences of centers forms a set of linearly independent vectors. Indeed, bearing in mind that the complement of every finite dimensional subspace of $X$ is dense in the infinite dimensional space $X$, we may inductively choose (taking $m$ points $y_{k}^{1}, \ldots, y_{k}^{m}$ at each $k$-th step of the induction process) sequences of points $\left(y_{n}^{j}\right)_{n=1}^{\infty}, j=1, \ldots, m$, with $y_{n}^{j} \in B\left(x_{n}^{j}, r_{n}^{j} / 2\right)$, so that:

(i) $\left\{y_{n}^{j}: n \in \mathbb{N}, j=1, \ldots, m\right\}$ is a set of linearly independent vectors;

(ii) $X=\bigcup_{n=1}^{\infty} B\left(y_{n}^{j}, r_{n}^{j}\right)$ for every $j=1, \ldots, m$; and

(iii) $\left|f^{j}(y)-f^{j}\left(y_{n}^{j}\right)\right| \leq \varepsilon_{j} / 4$ whenever $\left\|y-y_{n}^{j}\right\| \leq r_{n}^{j}$.

Next, for each collection of balls $\left\{B\left(y_{n}^{j}, r_{n}^{j}\right)\right\}_{n \in \mathbb{N}}$ and each function $f^{j}$, define scalloped balls $B_{n}^{j}$ and construct a function $\varphi^{j}$ exactly as in Case I above, so that

$$
\left|\varphi^{j}(y)-f^{j}(y)\right| \leq \varepsilon_{j} \quad \text { for all } \quad y \in B\left(y_{n}^{j}, r_{n}^{j}\right) .
$$


This function $\varphi^{j}$ is of the form

$$
\varphi^{j}(x)=\frac{\sum_{n=1}^{\infty} \alpha_{n}^{j}(x) \varphi_{n}^{j}(x)}{\sum_{n=1}^{\infty} \varphi_{n}^{j}(x)}=\lim _{n \rightarrow \infty} f_{n}^{j}(x),
$$

where

$$
f_{n}^{j}(x)=\frac{\sum_{k=1}^{n} \alpha_{k}^{j}(x) \varphi_{k}^{j}(x)}{\sum_{k=1}^{n} \varphi_{k}^{j}(x)}, \text { for all } x \in \bigcup_{i=1}^{n} B_{i}^{j},
$$

the domains of the $f_{n}^{j}$ form increasing towers of open sets whose union is $X$, and, for each $x \in X$ there is some open neighborhood $V_{x}^{j}$ of $x$ and some $n_{x}^{j} \in \mathbb{N}$ so that $\varphi^{j}(y)=f_{n}^{j}(y)$ for all $y \in V_{x}^{j}$ and all $n \geq n_{x}^{j}$ (see Fact 2.6).

Now define the mappings $\varphi: X \longrightarrow \mathbb{R}^{m}$ and $f_{n}: \bigcap_{j=1}^{m} \bigcup_{i=1}^{n} B_{i}^{j} \longrightarrow \mathbb{R}^{m}$ by

$$
\varphi(x)=\left(\varphi^{1}(x), \ldots, \varphi^{m}(x)\right), \text { and } f_{n}(x)=\left(f_{n}^{1}(x), \ldots, f_{n}^{m}(x)\right) .
$$

By the choice of the $\varepsilon_{j}$ and the construction of the functions $\varphi^{j}$, it is clear that

$$
\|\varphi(x)-f(x)\| \leq \varepsilon / 2, \text { for all } x \in X,
$$

that is, $\varphi$ approximates $f$ as is required.

Fact 2.10. If $x \in C_{f_{n}} \cap\left[\bigcap_{j=1}^{m} \bigcup_{i=1}^{n} B_{i}^{j}\right]$ then $x \in \mathcal{A}\left[y_{i}^{j}: 1 \leq i \leq n, 1 \leq j \leq m\right]$.

Proof. This is a consequence of Fact 2.8. Indeed, according to Remark 2.9, each $d f_{n}^{j}(x)$ is a nontrivial linear combination of the vectors $\left(x-y_{k}^{j}\right)($ with $k=1, \ldots, n)$. So, for each $j$ and each $x \in \bigcup_{i=1}^{n} B_{i}^{j}$ we can assign numbers $\beta_{1}^{j}(x), \ldots, \beta_{n}^{j}(x)$ such that at least one of them does not vanish, and

$$
d f_{n}^{j}(x)=\sum_{k=1}^{n} \beta_{k}^{j}(x)\left(x-y_{k}^{j}\right) .
$$

Suppose now that $x \in \bigcap_{j=1}^{m} \bigcup_{i=1}^{n} B_{i}^{j}$ and that the linear map $d f_{n}(x): X \longrightarrow \mathbb{R}^{m}$ is not surjective (that is, $x$ is a critical point of $f_{n}$ ); this means that there are numbers $\gamma_{1}(x), \ldots, \gamma_{m}(x)$, not all of them zero, such that

$$
\sum_{j=1}^{m} \gamma_{j}(x) d f_{n}^{j}(x)=0 .
$$

Then, by combining (12) and (13) we get that

$$
\sum_{j=1}^{m} \sum_{k=1}^{n} \gamma_{j}(x) \beta_{k}^{j}(x)\left(x-y_{k}^{j}\right)=0,
$$

where not all of the numbers $\gamma_{j}(x) \beta_{k}^{j}(x)$ vanish. Since the vectors $y_{k}^{j}$ are all linearly independent, it follows from (14) that $x$ is in the affine span of the vectors $y_{k}^{j}$ with $j=1, \ldots, m ; k=1, \ldots, n$. 
As $d \varphi$ is continuous, it is obvious that the set of critical points $C_{\varphi}$ is closed in $U$. Now we can easily show that $C_{\varphi}$ is locally compact as well. Indeed, take $x \in X$. For every $j=1, \ldots, m$ we know that there exists a neighborhood $V_{x}^{j}$ of $x$ and some $n_{x}^{j} \in \mathbb{N}$ so that $\varphi^{j}(y)=f_{n}^{j}(y)$ for all $y \in V_{x}^{j}$ and every $n \geq n_{x}^{j}$. Fix $n=n_{x}:=\max \left\{n_{x}^{1}, \ldots, n_{x}^{m}\right\}$, and take $W_{x}$ an open bounded neighborhood of $x$ so that $\overline{W_{x}} \subset V_{x}:=\bigcap_{j=1}^{m} V_{x}^{j}$. Then we have that

$$
\varphi(y)=\left(\varphi^{1}(y), \ldots, \varphi^{m}(y)\right)=\left(f_{n}^{1}(y), \ldots, f_{n}^{m}(y)\right)=f_{n}(y)
$$

for all $y \in V_{x}$, and in particular $V_{x} \subset \bigcap_{j=1}^{m} \bigcup_{i=1}^{n} B_{i}^{j}$. Now, according to Fact 2.10, it follows that $C_{\varphi} \cap V_{x}=C_{f_{n}} \cap V_{x}$ is contained in an affine subspace of dimension $n m$. In particular $C_{\varphi} \cap \overline{W_{x}}$ is compact, because it is closed, bounded, and is contained in a finite-dimensional subspace.

Remark 2.11. Let us say a few words as to the way one has to modify the above proofs in order to establish Proposition 2.1 when $\varepsilon$ is a continuous positive function. At the beginning of the proof of Case I of Proposition 2.1, before choosing the $\delta_{x}$, we have to take some number $\alpha_{x}>0$ so that $|\varepsilon(y)-\varepsilon(x)| \leq \varepsilon(x) / 4$ whenever $\|y-x\| \leq 2 \alpha_{x}$ and then we can find some $\delta_{x} \leq \alpha_{x}$ so that $|f(y)-f(x)| \leq \varepsilon(x) / 8$ whenever $y \in B\left(x, 2 \delta_{x}\right)$. In particular, after choosing the $r_{n}=\delta_{x_{n}}$ as in the proof of Case I above, we have that

$$
\left|f(y)-f\left(y_{n}\right)\right| \leq \varepsilon\left(y_{n}\right) / 8, \text { and } \varepsilon\left(y_{n}\right) \leq \frac{4}{3} \varepsilon(y)
$$

for all $y \in B\left(y_{n}, r_{n}\right)$. Then we can go on with the proof, with appropriate modifications, to construct the functions $\varphi$ and $f_{n}$. Some obvious changes must be made in the definition of the functions $a_{n}$ and $\alpha_{n}$. Fact 2.5 now tells us that

$$
\left|\varphi(y)-f\left(y_{n}\right)\right| \leq \varepsilon\left(y_{n}\right) / 4
$$

for all $y \in B\left(y_{n}, r_{n}\right)$. Then, by combining (15) and (16) we get that

$$
|\varphi(y)-f(y)| \leq\left|\varphi(y)-f\left(y_{n}\right)\right|+\left|f\left(y_{n}\right)-f(y)\right| \leq \frac{\varepsilon\left(y_{n}\right)}{4}+\frac{\varepsilon\left(y_{n}\right)}{8}=\frac{3}{8} \varepsilon\left(y_{n}\right) \leq \frac{\varepsilon(y)}{2}
$$

for all $y \in B\left(y_{n}, r_{n}\right)$ and, since these balls cover $X$, this proves that $|\varphi(y)-f(y)| \leq$ $\varepsilon(y) / 2$ for all $y \in X$.

In Case II it is enough to define the functions $\varepsilon_{j}(x)=\varepsilon(x) / \sqrt{4 m}$, for $j=1, \ldots, m$. The rest of the proof applies just replacing $\varepsilon_{j}$ and $\varepsilon$ with $\varepsilon_{j}(x)$ and $\varepsilon(x)$, and making some obvious minor modifications as in Case I.

\section{Proof of Theorem 2.2}

The proof of this result is based on that of Case I of Proposition 2.1. We will have to select the numbers $\lambda_{n}$ with more care, and make sure that the boundaries of the balls considered have a nice transversality property. An argument similar to that of Remark 2.11 shows that there is no loss of generality in assuming that $\varepsilon$ is constant. 
Suppose that we are at the beginning of the proof of Proposition 2.1 and we only know that $X=\bigcup_{n=1}^{\infty} B\left(y_{n}, s_{n} / 2\right)$, where $s_{n}=\delta_{x_{n}}$, for some sequence of linearly independent vectors $\left(y_{n}\right)$, and

$$
\left|f(y)-f\left(y_{n}\right)\right| \leq \varepsilon / 4 \text { provided }\left\|y-y_{n}\right\| \leq \frac{3}{2} s_{n} .
$$

The following lemma shows that we can slightly move the radii $s_{n}$ so that, for any finite selection of centers $y_{n}$, the spheres that are the boundaries of the balls $B\left(y_{n}, s_{n}\right)$ have empty intersection with the affine subspace spanned by those centers.

Lemma 2.12. We can find a sequence of positive numbers $\left(r_{n}\right)$ with $s_{n} \leq r_{n} \leq \frac{3}{2} s_{n}$ so that, if we denote $S_{n}=\partial B\left(y_{n}, r_{n}\right)$ then,

(i) for each finite sequence of positive integers $k_{1}<k_{2}<\ldots<k_{m}$,

$$
\mathcal{A}\left[y_{k_{1}}, \ldots, y_{k_{m}}\right] \cap S_{k_{1}} \cap \ldots \cap S_{k_{m}}=\emptyset .
$$

(ii) for any $n, k \in \mathbb{N}, y_{n} \notin S_{k}$.

Proof. We will define the $r_{n}$ inductively.

For $n=1$ we may take $r_{1} \in\left[s_{1}, \frac{3}{2} s_{1}\right]$ so that $r_{1}$ does not belong to the countable set $\left\{\left\|y_{1}-y_{k}\right\|: k \in \mathbb{N}\right\}$; this means that $y_{k} \notin S_{1}$ for any $k \in \mathbb{N}$. On the other hand, it is obvious that $\left\{y_{1}\right\} \cap S_{1}=\emptyset$.

Assume now that $r_{1}, \ldots, r_{n}$ have already been chosen in such a way that the spheres $S_{1}, \ldots, S_{n}$ satisfy $(i)$ and $(i i)$, and let us see how we can find $r_{n+1}$. For any finite sequence of integers $0<k_{1}<\ldots<k_{j} \leq n+1$, let us denote

$$
\mathcal{A}_{k_{1}, \ldots, k_{j}}=\mathcal{A}\left[y_{k_{1}}, \ldots, y_{k_{j}}\right] \text {. }
$$

For simplicity, and up to a suitable translation (which obviously does not affect our problem), we may assume that $y_{n+1}=0$, so that $\mathcal{A}_{k_{1}, \ldots, k_{m}, n+1}$ is the $m$-dimensional vector subspace of $X$ spanned by $y_{k_{1}}, \ldots, y_{k_{m}}$. Now, for each finite sequence of integers $0<k_{1}<\ldots<k_{m} \leq n$, consider the map $F_{k_{1}, \ldots, k_{m}}: \mathcal{A}_{k_{1}, \ldots, k_{m}, n+1} \longrightarrow \mathbb{R}^{m}$ defined by

$$
F_{k_{1}, \ldots, k_{m}}(x)=\left(\left\|x-y_{k_{1}}\right\|^{2}-{r_{k_{1}}}^{2}, \ldots,\left\|x-y_{k_{m}}\right\|^{2}-{r_{k_{m}}}^{2}\right)
$$

Note that

$$
D F_{k_{1}, \ldots, k_{m}}(x)=\left(2\left(x-y_{k_{1}}\right), \ldots, 2\left(x-y_{k_{m}}\right)\right)
$$

and therefore $\operatorname{rank}\left(D F_{k_{1}, \ldots, k_{m}}(x)\right)<m$ if and only if $x \in \mathcal{A}_{k_{1}, \ldots, k_{m}}$. By the induction assumption we know that

$$
S_{k_{1}} \cap \ldots \cap S_{k_{m}} \cap \mathcal{A}_{k_{1}, \ldots, k_{m}}=\emptyset
$$

hence it is clear that $\operatorname{rank}\left(D F_{k_{1}, \ldots, k_{m}}(x)\right)=m$ for all $x \in S_{k_{1}} \cap \ldots \cap S_{k_{m}} \cap \mathcal{A}_{k_{1}, \ldots, k_{m}, n+1}$. This implies that

$$
M_{k_{1}, \ldots, k_{m}}:=S_{k_{1}} \cap \ldots \cap S_{k_{m}} \cap \mathcal{A}_{k_{1}, \ldots, k_{m}, n+1}
$$


is a compact $m-m=0$-dimensional submanifold of $\mathcal{A}_{k_{1}, \ldots, k_{m}, n+1}$, and in particular $M_{k_{1}, \ldots, k_{m}}$ consists of a finite number of points (in fact two points, but we do not need to know this). Therefore

$$
M=\bigcup M_{k_{1}, \ldots, k_{m}}
$$

(where the union is taken over all the finite sequences of integers $0<k_{1}<\ldots<$ $\left.k_{n} \leq n\right)$ is a finite set as well. Now we have that

$$
I:=\left[s_{n+1}, \frac{3}{2} s_{n+1}\right] \backslash\left(\{\|z\|: z \in M\} \cup\left\{\left\|y_{j}\right\|: j \in \mathbb{N}\right\}\right)
$$

is an uncountable subset of the real line, so we can find a number $r_{n+1} \in I$. With this choice it is clear that

$$
S_{k_{1}} \cap \ldots \cap S_{k_{m}} \cap S_{n+1} \cap \mathcal{A}_{k_{1}, \ldots, k_{m}, n+1}=M_{k_{1}, \ldots, k_{m}} \cap S_{n+1}=\emptyset
$$

for all finite sequences of integers $0<k_{1}<\ldots<k_{m}<n+1$, and also

$$
y_{j} \notin S_{n+1}=\partial B\left(0, r_{n+1}\right) \text { for all } j \in \mathbb{N} .
$$

Therefore the spheres $S_{1}, \ldots, S_{n}, S_{n+1}$ satisfy $(i)$ and $(i i)$ as well. By induction the sequence $\left(r_{n}\right)$ is thus well defined.

Now define $B_{n}, \varphi_{n}, \varphi, f_{n}$, as in Case I of the proof of Proposition 2.1. All the properties shown in the proof of 2.1 about the functions $f_{n}$ and $\varphi$ (in particular Facts 2.5 and 2.8) are independent of the way we may choose the numbers $\lambda_{j}$ in the definitions of $B_{j}$ and $\varphi_{j}$. Now we only have to see how we can select those numbers $\lambda_{j}$ so as to have more control over the set $C_{\varphi}$ of critical points of $\varphi$ and thus prove the statement of Theorem 2.2. We will define the numbers $\lambda_{n}$ and the open sets $U_{n}$ inductively.

First step. Define $\varphi_{1}$ as above and put $f_{1}(x)=\alpha_{1}(x)$ for all $x \in B_{1}=B\left(y_{1}, r_{1}\right)$. Set $\mu_{2}=1 / 2, K_{1}=C_{f_{1}} \cap B_{1}=\left\{y_{1}\right\}$, and $U_{1}=B\left(y_{1}, \mu_{2} r_{1}\right)$.

Second step. Fix $\lambda_{2} \in\left(\mu_{2}, 1\right)$, and define $B_{2}, \varphi_{2}$, and $f_{2}$ as above. According to Fact 2.8, we have that

$$
\begin{aligned}
& C_{f_{2}} \cap B_{2} \subset \mathcal{A}\left[y_{1}, y_{2}\right], \text { and } \\
& C_{f_{2}} \cap\left(B_{2} \backslash B_{1}\right) \subseteq \mathcal{A}\left[y_{2}\right] .
\end{aligned}
$$

We claim that there must exist some $\mu_{3} \in\left(\lambda_{2}, 1\right)$ so that $\overline{C_{f_{2}} \cap B_{2} \cap B_{1}} \subset B\left(y_{1}, \mu_{3} r_{1}\right)$. Otherwise there would exist a sequence $\left(x_{j}\right)$ in $C_{f_{2}} \cap B_{2} \cap B_{1}$ so that $\left\|x_{j}-y_{1}\right\|$ goes to $r_{1}$ as $j$ goes to $\infty$. Since $C_{f_{2}} \cap B_{2} \subset \mathcal{A}\left[y_{1}, y_{2}\right]$, we may assume, by compactness, that $x_{j}$ converges to some point $x_{0} \in \partial B\left(y_{1}, r_{1}\right)=S_{1}$. If $x_{0} \in B\left(y_{2}, r_{2}\right)$ then $f_{2}^{\prime}\left(x_{0}\right)=0$ (by continuity of $f_{2}^{\prime}$ ), and $x_{0} \neq y_{2}$ (because $y_{2} \notin S_{1}$ by ii) of Lemma 2.12), so

$$
f_{2}^{\prime}\left(x_{0}\right)=\alpha_{2}^{\prime}\left(x_{0}\right) \neq 0,
$$

a contradiction. Therefore it must be the case that $x_{0} \in \partial B\left(y_{2}, r_{2}\right)=S_{2}$. But then

$$
x_{0} \in S_{1} \cap S_{2} \cap \mathcal{A}\left[y_{1}, y_{2}\right],
$$

and this contradicts Lemma 2.12 . 
So let us take $\mu_{3} \in\left(\lambda_{2}, 1\right)$ such that $\overline{C_{f_{2}} \cap B_{2} \cap B_{1}} \subset B\left(y_{1}, \mu_{3} r_{1}\right)$. Choose also some $\nu_{2} \in\left(\mu_{2}, \lambda_{2}\right)$. In the case that $y_{2} \in B_{1}$, let us simply set

$$
\begin{aligned}
& U_{2}=B\left(y_{2}, r_{2}\right) \cap B\left(y_{1}, \mu_{3} r_{1}\right) \backslash \bar{B}\left(y_{1}, \nu_{2} r_{1}\right), \text { and } \\
& K_{2}=\overline{C_{f_{2}} \cap B_{2} \cap B_{1}} \subset U_{2} .
\end{aligned}
$$

In the case that $y_{2} \notin B_{1}$, find $\delta_{2} \in\left(0, \mu_{3} r_{2}\right)$ so that $B\left(y_{2}, \delta_{2}\right) \subset B_{2} \backslash \overline{B_{1}}$, and set

$$
\begin{aligned}
& U_{2}=\left[B\left(y_{2}, r_{2}\right) \cap B\left(y_{1}, \mu_{3} r_{1}\right) \backslash \bar{B}\left(y_{1}, \nu_{2} r_{1}\right)\right] \cup B\left(y_{2}, \delta_{2}\right), \text { and } \\
& K_{2}=\overline{C_{f_{2}} \cap B_{2} \cap B_{1} \cup\left\{y_{2}\right\} \subset U_{2} .}
\end{aligned}
$$

Clearly, we have that $C_{f_{2}} \subseteq K_{1} \cup K_{2}$, and $U_{1} \cap U_{2}=\emptyset$.

Third step. Now choose $\lambda_{3} \in\left(\mu_{3}, 1\right)$ with $\lambda_{3}>1-1 / 3$, and define $B_{3}, \varphi_{3}$, and $f_{3}$ as above. We have that $f_{3}$ and $f_{2}$ coincide on $\left(B_{1} \cup B_{2}\right) \backslash B_{3}$. On $B_{3}$, according to Fact 2.8, we know that

$$
\begin{aligned}
& C_{f_{3}} \cap B_{3} \cap B_{2} \cap B_{1} \subseteq \mathcal{A}\left[y_{1}, y_{2}, y_{3}\right] ; \\
& C_{f_{3}} \cap\left(B_{3} \cap B_{2} \backslash B_{1}\right) \subseteq \mathcal{A}\left[y_{2}, y_{3}\right], \text { and } C_{f_{3}} \cap\left(B_{3} \cap B_{1} \backslash B_{2}\right) \subseteq \mathcal{A}\left[y_{1}, y_{3}\right] ; \\
& C_{f_{3}} \cap\left(B_{3} \backslash\left(B_{1} \cup B_{2}\right)\right) \subseteq \mathcal{A}\left[y_{3}\right] .
\end{aligned}
$$

Again, there must be some $\mu_{4} \in\left(\lambda_{3}, 1\right)$ so that

$$
\overline{C_{f_{3}} \cap B_{3} \cap\left(B_{1} \cup B_{2}\right)} \subset B\left(y_{1}, \mu_{4} r_{1}\right) \cup B\left(y_{2}, \mu_{4} r_{2}\right) .
$$

Otherwise (bearing in mind the local compactness of $\mathcal{A}\left[y_{1}, y_{2}, y_{3}\right]$ ), there would exist a sequence $\left(x_{j}\right)$ in $C_{f_{3}} \cap B_{3} \cap\left(B_{1} \cup B_{2}\right)$ so that $\left(x_{j}\right)$ converges to some point $x_{0}$ and $\left(x_{j}\right)$ is not contained in $B\left(y_{1}, \mu_{4} r_{1}\right) \cup B\left(y_{2}, \mu_{4} r_{2}\right)$ for any $\mu_{4}<1$. Since a subsequence of $\left(x_{j}\right)$ must be contained in one of the sets listed in (17), we deduce that the limit point $x_{0}$ must belong to one of the following sets:

$$
\begin{aligned}
& S_{2} \cap S_{1} \cap \mathcal{A}\left[y_{1}, y_{2}, y_{3}\right] ; \\
& S_{2} \cap \mathcal{A}\left[y_{2}, y_{3}\right] \backslash B_{1} ; \\
& S_{1} \cap \mathcal{A}\left[y_{1}, y_{3}\right] \backslash B_{2},
\end{aligned}
$$

Now we have two cases: either $x_{0} \in B_{3}$, or $x \in \partial B_{3}$. If $x_{0} \in B_{3}$ then $f_{3}^{\prime}\left(x_{0}\right)=0$ (by continuity of $f_{3}^{\prime}$ ), and $x_{0} \neq y_{3}$ (because $y_{3} \notin S_{1} \cup S_{2}$ by (ii) of Lemma 2.12), so it follows that

$$
f_{3}^{\prime}\left(x_{0}\right)=\alpha_{3}^{\prime}\left(x_{0}\right) \neq 0,
$$

a contradiction. On the other hand, if $x_{0} \in \partial B_{3}$ then $x_{0} \in S_{3}$ as well, and now one of the following must hold:

$$
\begin{aligned}
& x_{0} \in S_{3} \cap S_{2} \cap S_{1} \cap \mathcal{A}\left[y_{1}, y_{2}, y_{3}\right] ; \\
& x_{0} \in S_{3} \cap S_{2} \cap \mathcal{A}\left[y_{2}, y_{3}\right] ; \\
& x_{0} \in S_{3} \cap S_{1} \cap \mathcal{A}\left[y_{1}, y_{3}\right],
\end{aligned}
$$

but in any case this contradicts Lemma 2.12.

Hence we can take $\mu_{4} \in\left(\lambda_{3}, 1\right)$ so that

$$
\overline{C_{f_{3}} \cap B_{3} \cap\left(B_{1} \cup B_{2}\right)} \subset B\left(y_{1}, \mu_{4} r_{1}\right) \cup B\left(y_{2}, \mu_{4} r_{2}\right) .
$$


Take $\nu_{3} \in\left(\mu_{3}, \lambda_{3}\right)$. Now two possibilities arise. If $y_{3} \in B_{1} \cup B_{2}$, let us define

$$
U_{3}=\left[B\left(y_{3}, r_{3}\right) \backslash \bigcup_{j=1}^{2} \bar{B}\left(y_{j}, \nu_{3} r_{j}\right)\right] \bigcap\left[\bigcup_{j=1}^{2} B\left(y_{j}, \mu_{4} r_{j}\right)\right],
$$

and

$$
K_{3}=\overline{C_{f_{3}} \cap B_{3} \cap\left(B_{1} \cup B_{2}\right)} \subset U_{3} .
$$

If $y_{3} \notin B_{1} \cup B_{2}$, since $y_{3} \notin S_{1} \cup S_{2}$ we can find $\delta_{3} \in\left(0, \mu_{4} r_{3}\right)$ so that $B\left(y_{3}, \delta_{3}\right) \subseteq$ $B_{3} \backslash\left(B_{1} \cup B_{2}\right)$, and then we can set

$$
U_{3}=\left[\left(B\left(y_{3}, r_{3}\right) \backslash \bigcup_{j=1}^{2} \bar{B}\left(y_{j}, \nu_{3} r_{j}\right)\right) \bigcap\left(\bigcup_{j=1}^{2} B\left(y_{j}, \mu_{4} r_{j}\right)\right)\right] \bigcup B\left(y_{3}, \delta_{3}\right),
$$

and

$$
K_{3}=\overline{\left[C_{f_{3}} \cap B_{3} \cap\left(B_{1} \cup B_{2}\right)\right] \cup\left\{y_{3}\right\}} \subset U_{3} .
$$

Notice that $U_{3}$ does not meet $U_{1}$ or $U_{2}$, and $C_{f_{3}} \subseteq K_{1} \cup K_{2} \cup K_{3}$.

N-th step. Suppose now that $\mu_{j}, \lambda_{j}, \nu_{j}, \varphi_{j}, B_{j}, f_{j}, K_{j}, U_{j}$ have already been fixed for $j=1, \ldots, n$ (and also $\mu_{n+1}$ has been chosen) in such a manner that $f_{j}$ agrees with $f_{j-1}$ on $\left(B_{1} \cup \ldots \cup B_{j-1}\right) \backslash B_{j}$, and $K_{j}$ and $U_{j}$ are of the form

$$
K_{j}=\overline{C_{f_{j}} \cap B_{j} \cap\left(B_{1} \cup \ldots \cup B_{j-1}\right)}
$$

and

$$
U_{j}=\left[B\left(y_{j}, r_{j}\right) \backslash\left(\bigcup_{i=1}^{j-1} \bar{B}\left(y_{i}, \nu_{j} r_{i}\right)\right)\right] \bigcap\left[\bigcup_{i=1}^{j-1} B\left(y_{i}, \mu_{j+1} r_{i}\right)\right]
$$

in the case that $y_{j} \in B_{1} \cup \ldots \cup B_{j-1}$, and are of this form plus $\left\{y_{j}\right\}$ and $B\left(y_{j}, \delta_{j}\right)$ respectively when $y_{j} \notin B_{1} \cup \ldots \cup B_{j-1}$; assume additionally that $U_{j} \cap U_{k}=\emptyset$ whenever $j \neq k$, that $C_{f_{j}} \subseteq \bigcup_{i=1}^{j} K_{i}$, and that $\lambda_{j}>1-1 / j$. Let us see how we can choose $\lambda_{n+1}, \mu_{n+2}, \nu_{n+1}, K_{n+1}$ and $U_{n+1}$ so that the extended bunch keeps the required properties.

Pick any $\lambda_{n+1} \in\left(\mu_{n+1}, 1\right)$ so that $\lambda_{n+1}>1-1 /(n+1)$, and define $\varphi_{n+1}, B_{n+1}$ and $f_{n+1}$ as above. We know that $f_{n+1}$ agrees with $f_{n}$ on the set $\left(B_{1} \cup \ldots \cup B_{n}\right) \backslash B_{n+1}$. On $B_{n+1}$, according to Fact 2.8, we have that

$$
C_{f_{n+1}} \cap\left(B_{n+1} \backslash \bigcup_{j=1}^{m} B_{k_{j}}\right) \subseteq \mathcal{A}\left[\left\{y_{1}, \ldots, y_{n+1}\right\} \backslash\left\{y_{k_{1}}, \ldots, y_{k_{m}}\right\}\right]
$$

for every finite sequence of integers $0<k_{1}<k_{2}<\ldots<k_{m}<n+1$.

We claim that there exists some $\mu_{n+2} \in\left(\lambda_{n+1}, 1\right)$ so that

$$
\overline{C_{f_{n+1}} \cap B_{n+1} \cap\left(B_{1} \cup \ldots \cup B_{n}\right)} \subseteq \bigcup_{i=1}^{n} B\left(y_{i}, \mu_{n+2} r_{i}\right) .
$$


Otherwise there would exist a finite (possibly empty!) sequence of integers $0<k_{1}<$ $k_{2}<\ldots<k_{m}<n+1$, and a sequence $\left(x_{j}\right)_{j=1}^{\infty}$ contained in

$$
\left[C_{f_{n+1}} \cap B_{n+1} \cap\left(\bigcap_{j=1}^{\ell} B_{i_{j}}\right)\right] \backslash\left(\bigcup_{j=1}^{m} B_{k_{j}}\right) \subseteq \mathcal{A}\left[y_{i_{1}}, \ldots, y_{i_{\ell}}, y_{n+1}\right]
$$

(where $i_{1}, \ldots, i_{\ell}$ are the positive integers less than or equal to $n$ that are left when we remove $\left.k_{1}, \ldots, k_{m}\right)$, such that $\left(x_{j}\right)$ converges to some point $x_{0} \in S_{i_{1}} \cap \ldots \cap S_{i_{\ell}}$ with $x_{0} \notin \bigcup_{j=1}^{m} B_{k_{j}}$.

If $x_{0} \in B_{n+1}$ then $f_{n+1}^{\prime}\left(x_{0}\right)=0$ (by continuity of $f_{n+1}^{\prime}$ ), and $x_{0} \neq y_{n+1}$, so we easily see that

a contradiction.

$$
f_{n+1}^{\prime}\left(x_{0}\right)=\alpha_{n+1}^{\prime}\left(x_{0}\right) \neq 0,
$$

If $x_{0} \in \partial B_{n+1}$ then $x_{0} \in S_{n+1}$ as well, and in this case we have

$$
x_{0} \in S_{i_{1}} \cap \ldots \cap S_{i_{\ell}} \cap S_{n+1} \cap \mathcal{A}\left[y_{i_{1}}, \ldots, y_{i_{\ell}}, y_{n+1}\right],
$$

but this contradicts Lemma 2.12.

Therefore we may take $\mu_{n+2} \in\left(\lambda_{n+1}, 1\right)$ so that

$$
\overline{C_{f_{n+1}} \cap B_{n+1} \cap\left(B_{1} \cup \ldots \cup B_{n}\right)} \subseteq \bigcup_{i=1}^{n} B\left(y_{i}, \mu_{n+2} r_{i}\right) .
$$

Choose any $\nu_{n+1} \in\left(\mu_{n+1}, \lambda_{n+1}\right)$. As before, now we face two possibilities. If $y_{n+1} \in$ $\bigcup_{i=1}^{n} B_{i}$, let us define

$$
\begin{gathered}
U_{n+1}=\left[B\left(y_{n+1}, r_{n+1}\right) \backslash \bigcup_{i=1}^{n} \bar{B}\left(y_{i}, \nu_{n+1} r_{i}\right)\right] \cap\left[\bigcup_{i=1}^{n} B\left(y_{i}, \mu_{n+2} r_{i}\right)\right], \text { and } \\
K_{n+1}=\overline{C_{f_{n+1}} \cap B_{n+1} \cap\left(B_{1} \cup \ldots \cup B_{n}\right)} .
\end{gathered}
$$

If $y_{n+1} \notin \bigcup_{i=1}^{n} B_{i}$, since $y_{n+1} \notin S_{i}$ we may find $\delta_{n+1} \in\left(0, \mu_{n+2} r_{n+1}\right)$ so that $B\left(y_{n+1}, \delta_{n+1}\right) \subseteq B_{n+1} \backslash \bigcup_{i=1}^{n} B_{i}$, and then we can add this ball to the above $U_{n+1}$, and the point $\left\{y_{n+1}\right\}$ to that $K_{n+1}$, in order to obtain sets $U_{n+1}, K_{n+1}$ with the required properties.

By induction, the sequences $\left(\varphi_{n}\right),\left(f_{n}\right),\left(U_{n}\right),\left(K_{n}\right),\left(\lambda_{n}\right),\left(\mu_{n}\right),\left(\nu_{n}\right)$ are well defined and satisfy the above properties. From the construction it is clear that $U_{n} \cap U_{m}=\emptyset$ whenever $n \neq m$, and

$$
C_{f_{n}} \subseteq \bigcup_{j=1}^{n} K_{j}
$$

for all $n$. As observed before (see Fact 2.6), for each $x \in X$ there exists an open neighborhood $V_{x}$ of $x$ and some $n_{x} \in \mathbb{N}$ so that $\varphi(y)=f_{n_{x}}(y)$ for all $y \in V_{x}$. Bearing these facts in mind, it is immediately checked that $C_{\varphi} \subseteq \bigcup_{n=1}^{\infty} K_{n}$. The other properties in the statement of Theorem 2.2 are immediately deduced from the above construction. 


\section{Acknowledgements}

We are indebted to Tadeusz Dobrowolski, who read the first version of this paper and suggested a simplification of the original proof by pointing out to us West's paper [23]. We also wish to thank Pilar Cembranos, José Mendoza, Tijani Pakhrou and Raúl Romero, who helped us to realize that Fact 2.8 fails whenever the norm is not hilbertian. This research was in part carried out during a stay of the first-named author in the Mathematics Department of University College London; this author wish to thank the members of that Department and very especially David Preiss for their kind hospitality and advice.

\section{REFERENCES}

[1] D. Azagra and T. Dobrowolski, Smooth negligibility of compact sets in infinite-dimensional Banach spaces, with applications, Math. Ann. 312 (1998), no. 3, 445-463.

[2] D. Azagra and M. Jiménez-Sevilla, The failure of Rolle's Theorem in infinite dimensional Banach spaces, J. Funct. Anal. 182 (2001), 207-226.

[3] S. M. Bates, On the image size of singular maps. I., Proc. Amer. Math. Soc. 114 (1992), no. 3, 699-705.

[4] S. M. Bates, On the image size of singular maps. II., Duke Math. J. 68 (1992), no. 3, 463-476.

[5] S. M. Bates, Toward a precise smoothness hypothesis in Sard's theorem, Proc. Amer. Math. Soc. 117 (1993), no. 1, 279-283.

[6] S. M. Bates, On smooth rank-1 mappings of Banach spaces onto the plane, J. Differential Geom. 37 (1993), no. 3, 729-733.

[7] S. M. Bates, On smooth non-linear surjections of Banach spaces, Israel J. Math. 100 (1997), 209-220.

[8] S. M. Bates and C. G. Moreira, De nouvelles perspectives sur le théorème de Morse-Sard, C.R. Acad. Sci. Paris, t. 332, Série I (2001), p. 13-17.

[9] C. Bessaga, Every infinite-dimensional Hilbert space is diffeomorphic with its unit sphere, Bull. Acad. Polon. Sci., Sér. Sci. Math. Astr. et Phys.14 (1966), pp. 27-31.

[10] C. Bessaga and A. Pelczynski, Selected topics in infinite-dimensional topology, Monografie Matematyczne, Tom. 58 Polish Scientific Publishers, Warszawa, 1975.

[11] H. Cartan, Calcul différentiel, Hermann, Paris 1967.

[12] R. Deville, G. Godefroy, and V. Zizler, Smoothness and renormings in Banach spaces, vol. 64, Pitman Monographs and Surveys in Pure and Applied Mathematics, Longman Scientific \& Technical, Harlow, 1993.

[13] J. Eells and K.D. Elworthy, Open embeddings of certain Banach manifolds, Ann. of Math. 91 (1970), 465-485.

[14] J. Eells and J. McAlpin, An approximate Morse-Sard theorem, J. Math. Mech. 17 (1967/1968), 1055-1064.

[15] T. Dobrowolski, Smooth and R-analytic negligibility of subsets and extension of homeomorphism in Banach spaces, Studia Math. 65 (1979), 115-139.

[16] I. Kupka, Counterexample to the Morse-Sard theorem in the case of infinite-dimensional manifolds, Proc. Amer. Math. Soc. 16 (1965), 954-957.

[17] C. G. Moreira, Hausdorff measures and the Morse-Sard theorem, Publ. Mat. 45 (2001), 149-162.

[18] A. Morse, The behavior of a function on its critical set, Annals of Math. 40 (1939), 62-70.

[19] A. Sard, The measure of the critical values of differentiable maps, Bull. Amer. Math. Soc. 48 (1942), 883-890.

[20] A. Sard, Images of critical sets, Annals of Math. 68 (1958), 247-259.

[21] A. Sard, Hausdorff measure of critical images on Banach manifolds, Amer. J. Math. 87 (1965), 158-174. 
[22] S. Smale, An infinite dimensional version of Sard's theorem, Am. J. Math. 87 (1965), 861-866.

[23] J. E. West, The diffeomorphic excision of closed local compacta from infinite-dimensional Hilbert manifolds, Compositio Math. 21 (1969), 271-291.

[24] H. Whitney, A function not constant on a connected set of critical points, Duke Math. J. 1 (1935), 514-517.

Departamento de Análisis Matemático. Facultad de Ciencias Matemáticas. Universidad Complutense. 28040 Madrid, SPAIN

Departamento de Análisis Matemático. Universidad de Sevilla. Sevilla, SPAIN. E-mail addresses: daniel_azagra@mat.ucm.es, mcb@us.es 